\title{
Untersuchungen zum Substituenten- und Lösungsmitteleinfluß auf Solvolysereaktionen
}

\section{Der Einfluß polarer $\alpha$-Substituenten auf die Geschwindigkeit der alkalischen Esterhydrolyse in Wasser bei verschiedenen Temperaturen \\ Von}

\section{J. BARTHEL, G. BÄDER und G. SCHMEER}

Institut für physikalische Chemie der Universität des Saarlandes, Saarbrücken Herrn Professor B. Eistert zum 65. Geburtstag gewidmet

Mit 3 Abbildungen

(Eingegangen am 28. Februar 1968)

Aus der schnell verlaufenden Leitfähigkeitsänderung beim Reaktionsablauf werden nach einer früher beschriebenen Methode ${ }^{1}$ die Geschwindigkeitskonstanten und die Aktivierungsgrößen der Verseifung von Mono-, Di-, Trihalogen-, Hydroxy-, Methoxy- und Äthoxyessigsäuremethyl- und -äthylester bestimmt. Der Substituenteneinfluß wird aus der elektrostatischen Wechselwirkung der Reaktionspartner erklärt.

\section{Einführung}

Die Geschwindigkeitskonstante $k$ der alkalischen Hydrolyse eines substituierten Essigsäureesters in Wasser

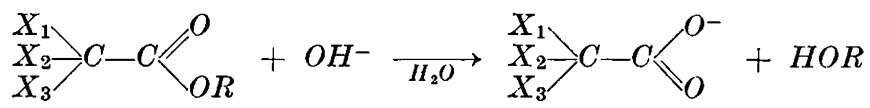

verschiebt sich gegenüber der Konstanten $k_{0}$ aus der Reaktion des entsprechenden unsubstituierten Esters $\left(X_{1}=X_{2}=X_{3}=H\right)$ gemäß

$$
\ln k-\ln k_{0}=\frac{1}{R T}\left(\Delta G_{0}^{\ddagger}-\Delta G^{\ddagger}\right) .
$$

$\Delta G^{\ddagger}$ ist als freie Aktivierungsenthalpie der reversiblen Arbeit gleich, die erforderlich ist, um den Zwischenkomplex aus den Ausgangs-

1 J. Barthel und G. Bäder, Z. physik. Chem. Neue Folge 48 (1966) 109. 
produkten aufzubauen. Erzeugen die Reaktionspartner auf Grund ihrer inneren Ladungsverteilung (Einzelladungen oder Dipole) in ihrer Umgebung elektrostatische Felder, so ist der Anteil $\Delta G_{2}{ }^{\ddagger}$ in $\Delta G^{\ddagger}$, der aus ihrer elektrostatischen Wechselwirkung herrührt, berechenbar.

$$
\Delta G^{\ddagger}=\Delta G_{1}^{\ddagger}+\Delta G_{2}^{\ddagger} .
$$

Wir haben mit einer Reihe von $\alpha$-substituierten Essigsäureestern untersucht, inwieweit in $k$ und $k_{0}$ die Anteile $\Delta G_{1}^{\neq}$und $\Delta G_{01}^{\neq}$gleich sind, so daß Gleichung (1) als

$$
\ln k-\ln k_{0}=\frac{1}{R T}\left(\Delta G_{02}^{\ddagger}-\Delta G_{2}^{\ddagger}\right)=\frac{1}{R T} \Delta G_{e l}
$$

geschrieben und ( $\ln k-\ln k_{0}$ ) berechnet werden kann.

Die für eine solche Untersuchung notwendigen Geschwindigkeitskonstanten zusammen mit ihrer Temperaturabhängigkeit mußten neu, zum Teil erstmals, bestimmt werden. Die bisher bekannten Konstanten sind nach verschiedenen Methoden - potentiometrische Messung 2,3, Probenentnahme mit photometrischer Konzentrationsbestimmung ${ }^{4}$, Konzentrationsbestimmung mit Hochfrequenzoszillatoren ${ }^{5}, p H$-stationäre und coulometrische Messungen ${ }^{6}$ - mit erheblichen Abweichungen voneinander erhalten worden. Unsere Bestimmungen erfolgten erstmals aus Leitfähigkeitsmessungen. Meßanordnung und Meßmethode sind in loc. cit. ${ }^{1}$ beschrieben. Abweichend davon wurde die Leitfähigkeitsmeßzelle (loc. cit.1 Abb.1) ganz aus Polypropylen hergestellt. Diese Maßnahme war wegen der geringen Konzentration der eingesetzten Basen (bis zu $10^{-5}[\mathrm{Mol} / \mathrm{l}]$ ) erforderlich, da in Glaszellen die Reaktion der Basen mit den Glasoberflächen die Geschwindigkeitskonstanten der Esterhydrolyse systematisch verfälscht.

\section{Meßergebnisse}

Die Reaktionen zwischen den sorgfältig gereinigten Reaktionspartnern liefen unter sauerstoff- und kohlendioxidfreiem Stickstoff ab.

Die Ester waren Produkte vom höchstmöglichen, handelsüblichen Reinheitsgrad und wurden nochmals durch fraktionierte Destillation unter vermindertem Druck gereinigt. Thr Reinheitsgrad wurde über den Siedepunkt $K p$ und den

2 G. J. Nolan und E. S. Amis, J. physic. Chem. 65 (1961) 1556.

${ }^{3}$ R. P. Bell und J. E. Prue, Trans. Faraday Soc. 46 (1950) 14.

4 W. P. Jencks und J. Carriuolo, J. Amer. chem. Soc. 83 (1961) 1743.

5 D. G. Flom und P. J. Elving, Analyt. Chem. 25 (1953) 541.

6 E. K. Euranto und A. L. Moisio, Suomen Kemistilehti 37 B (1964) 92. 
Brechungsindex $n_{D}$ kontrolliert (vgl. Tab. 1). Die Base - NaOH - wurde mit den üblichen Vorsichtsmaßnahmen in sauerstoff- und kohlendioxidfreiem Leitfähigkeitswasser hergestellt und unter gereinigtem Stickstoff in einem Polypropylengefäß aufbewahrt, aus dem unter Stickstoffüberdruck jeweils 50 bis 100 [ml] in die Meßzelle eingeschleust wurden. Die eingesetzte Menge an Lösung wurde ausgewogen. Der Ester wurde in alkoholischer Lösung (Verdünnung bis zu 1:100) nach Erreichen des thermischen Gleichgewichtes (Temperaturkonstanz $\pm 0,002^{\circ}$ in der Meßzelle) mit einer HammLton-Spritze (10 bis $20[\mu l]$ ) zugegeben. Die Vermischungszeit war in jedem Fall kleiner als eine Halbwerts. zeit der Reaktion.

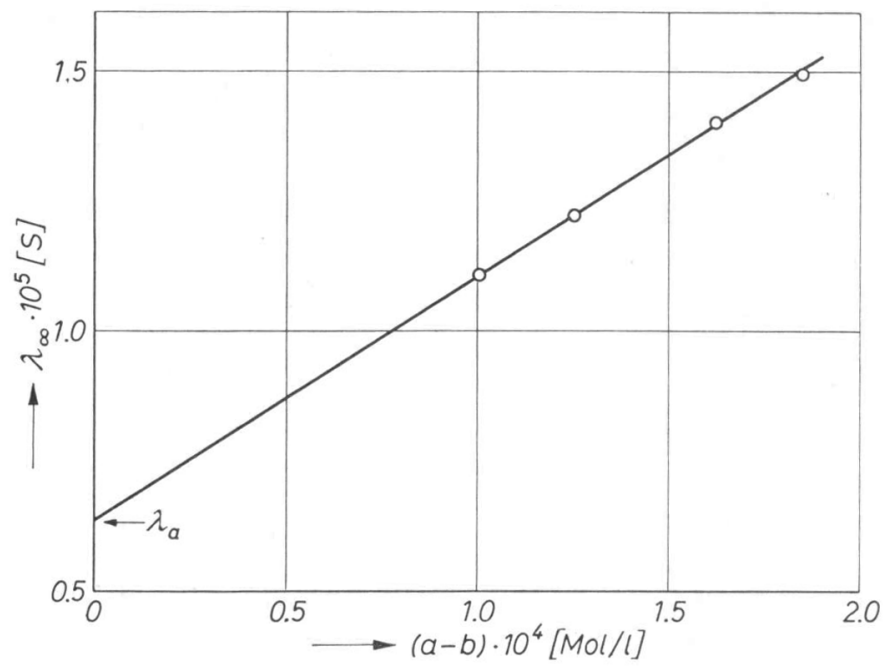

Abb.1. Bestimmung des Wertes $R_{a}$ durch Extrapolation gemäß Gleichung (4) für die Reaktion $\mathrm{F}_{2} \mathrm{CH}-\mathrm{CO}_{2} \mathrm{C}_{2} \mathrm{H}_{5}+\mathrm{OH}^{-}$bei $25^{\circ} \mathrm{C}$

Die Registrierung des Reaktionsablaufes, ebenso wie die Auswertung der Daten, erfolgte nach den in loc. cit. ${ }^{1}$ angegebenen Methoden. Die Geschwindigkeitskonstante wird aus dem Anstieg der Funktion

$$
\log \frac{R_{a}-R_{(t)}}{R_{\infty}-R_{(t)}}=0,4343(a-b) k \cdot t+\text { Const., } a>b
$$

( $a$ und $b$ Anfangskonzentrationen von Base und Ester, $R_{(t)}$ Widerstandswert zum Zeitpunkt $t, R_{\infty}$ und $R_{a}$ Widerstandswerte nach vollständiger Reaktion des Esters bzw. der Base) erhalten.

Bei Einsatz einer $10^{-5} N$ Natronlauge und $5 \cdot 10^{-6}[\mathrm{Mol} / \mathrm{l}]$ Ester wird die Grenze für die Anwendung einer konduktometrischen Titration zur Bestimmung von $(a-b)$ erreicht. Man kann in diesem Fall $R_{a}$ immer noch mit ausreichender Genauigkeit aus der Titrationskurve, $(a-b)$ jedoch nur aus der genauen Einwaage der Reaktions- 
Tabelle 1. Brechungsindizes $n_{D}^{20}$ und Geschwindigkeitskonstanten $k$ in $[l / M o l \cdot \min ]$

\begin{tabular}{|c|c|c|c|c|c|}
\hline \multirow{2}{*}{$\begin{array}{c}\text { Ester } \\
R \mathrm{CO}_{2} R^{\prime}\end{array}$} & \multirow{2}{*}{$\begin{array}{l}\text { Brechungs- } \\
\text { index } n_{D}^{20}\end{array}$} & \multicolumn{4}{|c|}{ Geschwindigkeitskonstanten $k[\mathrm{l} / \mathrm{Mol} \cdot \min ]$} \\
\hline & & $15^{\circ} \mathrm{C}$ & $25^{\circ} \mathrm{C}$ & $30^{\circ} \mathrm{C}$ & $35^{\circ} \mathrm{C}$ \\
\hline \multicolumn{6}{|l|}{$\begin{array}{l}R^{\prime}=C H_{3} \\
R\end{array}$} \\
\hline $\mathrm{CH}_{3}$ & 1,3622 & & 11,45 & 16,05 & 22,25 \\
\hline $\mathrm{CH}_{3} \mathrm{OCH}_{2}$ & 1,3965 & & 174,5 & 235,7 & 305,5 \\
\hline $\begin{array}{l}\mathrm{ClCH}_{2} \\
\mathrm{Cl}_{2} \mathrm{CH} \\
\mathrm{Cl}_{3} \mathrm{C}\end{array}$ & $\begin{array}{l}1,4219 \\
1,4428 \\
1,4571\end{array}$ & $\begin{array}{r}1750 \\
44000 \\
141000\end{array}$ & $\begin{array}{r}3080 \\
73000 \\
223000\end{array}$ & & $\begin{array}{r}5300 \\
110000 \\
342000\end{array}$ \\
\hline \multicolumn{6}{|l|}{$\begin{array}{l}R^{\prime}=C_{2} H_{5} \\
R\end{array}$} \\
\hline $\mathrm{CH}_{3}$ & 1,3727 & 3,51 & 6,71 & & 13,40 \\
\hline $\begin{array}{l}\mathrm{HOCH}_{2} \\
\mathrm{CH}_{3} \mathrm{OCH} \mathrm{CH}_{2} \\
\mathrm{C}_{2} \mathrm{H}_{5} \mathrm{OCH} \mathrm{CH}_{2}\end{array}$ & $\begin{array}{l}1,4156 \\
1,4031 \\
1,3995\end{array}$ & $\begin{array}{l}39,9 \\
50,6\end{array}$ & $\begin{array}{l}76,0 \\
90,3 \\
70,8\end{array}$ & $\begin{array}{r}104,0 \\
119,3 \\
93,4\end{array}$ & $\begin{array}{l}142,6 \\
153,4 \\
122,0\end{array}$ \\
\hline $\begin{array}{l}\mathrm{FCH}_{2} \\
\mathrm{~F}_{2} \mathrm{CH} \\
\mathrm{F}_{3} \mathrm{C}\end{array}$ & $\begin{array}{l}1,3759 \\
1,3469 \\
1,3095\end{array}$ & $\begin{array}{r}503 \\
109000 \\
682000 \\
858000\end{array}$ & $\begin{array}{r}921 \\
176000 \\
1102000 \\
\end{array}$ & 1406000 & $\begin{array}{r}1597 \\
266000\end{array}$ \\
\hline $\begin{array}{l}\mathrm{ClCH}_{2} \\
\mathrm{Cl}_{2} \mathrm{CH} \\
\mathrm{Cl}_{3} \mathrm{C}\end{array}$ & $\begin{array}{l}1,4210 \\
1,4385 \\
1,4505\end{array}$ & $\begin{array}{r}1010 \\
21100 \\
77700\end{array}$ & $\begin{array}{r}1690 \\
32300 \\
120000\end{array}$ & & $\begin{array}{r}2890 \\
49000 \\
180000\end{array}$ \\
\hline $\mathrm{BrCH}_{2}$ & 1,4491 & 1360 & 2240 & & 3710 \\
\hline $\mathrm{JCH}_{2}$ & 1,5051 & 558 & 894 & & 1480 \\
\hline
\end{tabular}

partner erhalten. Die Bestimmung des $R_{a}$-Wertes wurde in einigen Fällen nach einer anderen Methode vorgenommen. Wählt man in einer Versuchsreihe bei variabler Ausgangskonzentration (b) an Ester stets die gleiche Ausgangskonzentration $(a)$ an Base, so ergibt sich aus der Auftragung $1 / R_{\infty}=f(a-b)$ der Wert $1 / R_{a}$ für alle Reaktionen aus der Extrapolation $a=b$ gemäß (vgl. Abb.1)

$$
1 / R_{\infty}-1 / R_{a}=\alpha(a-b)
$$

( $\alpha=$ Proportionalitätsfaktor). 
Die Geschwindigkeitskonstanten der untersuchten Hydrolysereaktionen sind in Tab. 1 angegeben. Die mittleren Fehler betragen für die Konstanten unter $k=50[\mathrm{l} / \mathrm{Mol} \cdot \mathrm{min}]$ weniger als $1 \%$ und steigen bis zur Größe von $4 \%$ für $k=10^{6}[1 / \mathrm{Mol} \cdot \min ]$.

Die Geschwindigkeitskonstanten sind sowohl von den Anfangskonzentrationen $a$ und $b$ als auch vom Verhältnis $b / a$ unabhängig. Der Mittelwert ist aus Konstanten gebildet, bei denen dieses Verhältnis in den Grenzen $0,2 \leqslant b / a \leqslant 0,5$ liegt.

\section{Theoretische Grundlagen}

Für den Zwischenkomplex der Reaktion wird ein Modell, wie es am Beispiel Abb. 2 dargestellt ist, angenommen. Die drei SauerstoffKohlenstoffbindungen haben zusammen mit der $C-C$-Bindung

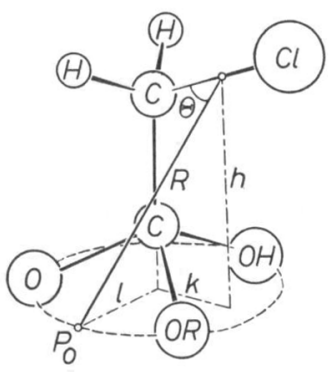

Abb.2. Struktur des Übergangskomplexes für die Reaktion

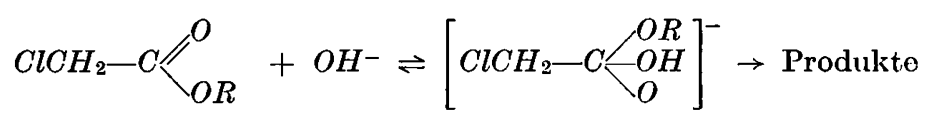

tetraedrische Anordnung; die negative Ladung wird gleichmäßig auf einen Kreis durch die drei Sauerstoffatome der Estergruppe verteilt?. Für die $C-C$-Bindung wird freie Drehbarkeit angenommen.

Der elektrostatische Anteil $\Delta G_{2}{ }^{\ddagger}$ der molaren freien Aktivierungsenthalpie ist ( $N=$ Loschmidt-Zahl)

$$
\Delta G^{\ddagger}=N \cdot e_{0} \cdot z \cdot \overline{U\left(P_{0}\right)},
$$

wenn ein Ion der Ladung $e_{0} \cdot z$ (hier $O H^{-}$mit $z=-1$ ) zum Aufbau des Zwischenkomplexes in $P_{0}$ angelagert wird. $U\left(P_{0}\right)$ ist das von der Ladungsverteilung des Estermoleküls im Zwischenkomplex an dieser Stelle erzeugte Potential, $\overline{U\left(P_{0}\right)}$ sein Mittelwert über alle Konfigurationen, die bei freier Drehbarkeit der $C$-C-Bindung mit gleichem statistischem Gewicht angenommen werden können. Zur Berechnung

7 R. P. Bell und B. A. W. Coller, Trans. Faraday Soc. 61 (1965) 1445. 
von $U(P)=U(r, \vartheta, \varphi)$ wird die Ladungsverteilung Abb.2 in eine dielektrische Kugel $\left(\varepsilon_{1}=1\right.$, Radius $\left.a\right)$ gebettet. Das Lösungsmittel umgibt diese Kugel als homogenes, isotropes Medium der $D K \varepsilon_{2}=\varepsilon$. Dann lassen sich durch Integration der PoIsson-Gleichungen

$$
\begin{array}{lll}
\Delta U_{1}=0 & \text { im ladungsfreien Gebiet } & r \leqslant a \\
\Delta U_{2}=0 & \text { für } \quad r \geqslant a
\end{array}
$$

die Potentiale $U_{1}(r, \vartheta, \varphi)$ und $U_{2}(r, \vartheta, \varphi)$ als Entwicklungen nach den Kugelfunktionen $P_{j}^{(m)}(\cos \vartheta)$ mit den Koeffizienten $A_{j}{ }^{(m)}, B_{j}{ }^{(m)}, C_{j}(m)$ und $D_{j}^{(m)}$ schreiben:

$$
\begin{gathered}
U_{1}(r, \vartheta, \varphi)=\sum_{j=0}^{\infty} \sum_{m=-j}^{j}\left[A_{j}(m) \cdot r^{j}+B_{j}(m) \cdot r^{-(j+1)}\right] P_{j}(m)(\cos \vartheta) e^{i m \varphi}(6 \mathrm{a}) \\
\text { im ladungsfreien Gebiet } r \leqslant a, \\
U_{2}(r, \vartheta, \varphi)=\sum_{j=0}^{\infty} \sum_{m=-j}^{j}\left[C_{j}(m) \cdot r^{j}+D_{j}(m) \cdot r^{-(j+1)}\right] P_{j}^{(m)}(\cos \vartheta) e^{i m \varphi}(6 \mathrm{~b}) \\
\text { im Gebiet } r \geqslant a .
\end{gathered}
$$

Die Koeffizienten der Reihen (6a) und (6b) sind durch die Randwerte

$$
\begin{aligned}
& \lim _{r \rightarrow \infty} U_{2}(r, \vartheta, \varphi)=0 \\
& U_{1(r=a)}=U_{2(r=a)} \\
& \left(\frac{\partial U_{1}}{\partial r}\right)_{r=a}=\varepsilon\left(\frac{\partial U_{2}}{\partial r}\right)_{r=a}
\end{aligned}
$$

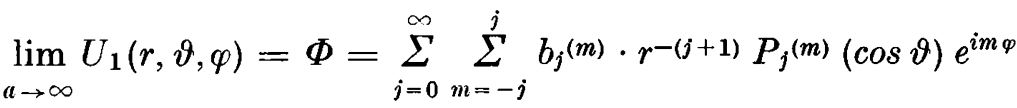

bestimmt. $\Phi(r, \vartheta, \varphi)$ ist das im Zwischenkomplex im Vakuum von der isolierten Ladungsverteilung des Estermoleküls erzeugte Potential, seine Koeffizienten $b_{j}{ }^{(m)}$ sind aus den Lagekoordinaten der Ladungen in bezug auf das vorgegebene Koordinatensystem eindeutig festgelegt. Unter Berücksichtigung von (7a) bis (7d) folgt aus (6a) und (6b)

$$
\begin{gathered}
U_{1}=\sum_{j=0}^{\infty} \sum_{m=-j}^{j} b_{j}(m)\left[-\frac{(\varepsilon-1)(j+1)}{\varepsilon(j+1)+j} \cdot \frac{r^{j}}{a^{2 j+1}}+\frac{1}{r^{j+1}}\right] P_{j}^{(m)}(\cos \vartheta) e^{i m \varphi} \\
U_{2}=\sum_{j=0}^{\infty} \sum_{m=-j}^{j} b_{j}^{(m)}\left[\frac{2 j+1}{\varepsilon(j+1)+j} \cdot \frac{1}{r^{j+1}}\right] P_{j}^{(m)}(\cos \vartheta) e^{i m \varphi} .
\end{gathered}
$$

Will man das Reaktionsfeld ${ }^{8} \vec{Z}$, das aus der Wechselwirkung des Zwischenkomplexes in der Kugel mit dem Lösungsmittel resultiert,

${ }^{8}$ C. J. F. Bötтcher, Theory of Electric Polarisation. Amsterdam 1952. 
in die Überlegungen einbeziehen, so kann dieses unter den üblichen Bedingungen gemäß

$$
\vec{Z}=-\frac{3}{4 \pi a^{3}} \iint_{r<a} \overrightarrow{\operatorname{grad}}\left[U_{1}(r, \vartheta, \varphi)-\Phi(r, \vartheta, \varphi)\right] d V
$$

bestimmt werden.

\section{Diskussion der Ergebnisse}

Zur Berechnung von $\Delta G_{e l}$ müssen die Koeffizienten $b_{0 j}^{(m)}$ und $b_{j}^{(m)}$ für den nicht substituierten bzw. substituierten Ester, die miteinander verglichen werden sollen, nicht vollständig bestimmt werden. Für die Differenz $\left(\Delta G_{02}^{\ddagger}-\Delta G_{2}^{\ddagger}\right)$ unterscheiden sich die Ladungsverteilungen nur um die Dipole der Substituenten und der durch sie ersetzten $C-H$-Bindungen. Den durch Verschiedenheit der Substituenten bedingten induzierten Momenten in den übrigen Bindungen muß dabei Rechnung getragen werden.

\section{a) Einfach substituierte Ester}

Für einfach substituierte Ester (vgl. Abb.2) ergibt sich eine wesentliche Vereinfachung der Gleichungen (8a) und (8b), wenn der Zwischenkomplex in der Kugel $r=a$ so angeordnet wird, daß der Dipol des Substituenten in den Koordinatenursprung zu liegen kommt und die $z$-Achse mit der Dipolausrichtung zusammenfällt. Dies bedeutet keine Einschränkung der Gültigkeit der Überlegungen, da die Wechselwirkung in jedem Falle als Funktion der Lagekoordinaten $(R, \theta)$ von $P_{0}$ in bezug auf den Substituentendipol ausgedrückt wird. Die geänderte Raumausfüllung in der dielektrischen Kugel durch das Estermolekül drückt sich über die Radien $a$ und $R$ in der durch das Modell postulierten $D K$-Abhängigkeit der Geschwindigkeitskonstanten aus.

Da der Punkt $P_{0}$ auf der Kugel $r=a$ oder innerhalb derselben liegt, genügt für die weitere Diskussion die Berücksichtigung von $U_{1}(P)=U(P)$.

$$
\begin{aligned}
U(P) & =\left[1-\frac{2 \varepsilon-2}{2 \varepsilon+1} \cdot \frac{r^{3}}{a^{3}}\right] \cdot \frac{\mu}{r^{2}} \cos \vartheta \\
& +\sum_{j=0}^{\infty} \sum_{m=-j}^{j} b^{\prime}(m)\left[-\frac{(\varepsilon-1)(j+1)}{\varepsilon(j+1)+j} \cdot \frac{r^{j}}{a^{2 j+1}}+\frac{1}{r^{j+1}}\right] P_{j}(m)(\cos \vartheta) e^{i m \varphi}
\end{aligned}
$$


oder

$$
U(P)=\left[1-\frac{2 \varepsilon-2}{2 \varepsilon+1} \cdot \frac{r^{3}}{a^{3}}\right] \frac{\mu}{r^{2}} \cos \vartheta+U^{\prime}(r, \vartheta, \varphi) .
$$

Die $b_{j}{ }^{\prime}(m)$ sind die neuen Koeffizienten der Entwicklung (8a) mit Ausnahme des Anteiles $\mu$ aus $b_{1}^{\prime(0)}$, der als Moment des Substituentendipols aus der Summe herausgenommen worden ist.

In der Differenz [vgl. Gleichungen (2) und (5)]

$$
\Delta G_{e l}=N \cdot e_{0} \cdot z\left[\overline{U_{0}\left(P_{0}\right)}-\overline{U\left(P_{0}\right)}\right]
$$

sind die beiden gemäß Gleichung (11) definierten Terme der Entwicklung $U_{0}{ }^{\prime}$ und $U^{\prime}$ für den nicht substituierten Bezugsester und den substituierten Ester näherungsweise gleich:

$$
U_{0}{ }^{\prime}-U^{\prime} \approx 0,
$$

da sie die für beide Ester gleichen Anteile der Ladungsverteilung des Restmoleküls sowie die höheren Terme des Dipolfeldes von $C-H$ bzw. des Substituenten beinhalten.

Dann folgt aus (2), (11) und (12)

$$
\ln k-\ln k_{0}=\frac{e_{0} \cdot z}{k T} \cdot F(\varepsilon)\left[\mu_{0} \cdot \overline{\cos \theta_{0} / R_{0}^{2}}-\mu \cdot \overline{\cos \theta / R^{2}}\right],
$$

wobei die Lage $P_{0}$ des Hydroxylions im Zwischenkomplex nunmehr durch die Koordinaten $\vartheta=\theta$ und $r=R$ festgelegt ist; $\mu_{0}$ bedeutet gemäß Gleichung (12) das Moment des $C-H$-Dipols, $\mu$ das des Substituenten.

Die Funktion

$$
F(\varepsilon)=\left(1-\frac{2 \varepsilon-2}{2 \varepsilon+1} \cdot \frac{\overline{R^{3}}}{a^{3}}\right)
$$

stellt die vom Modell geforderte Abhängigkeit von der Dielektrizitätskonstanten des Lösungsmittels dar. Sie verhält sich für $R=a$ wie $1 /(2 \varepsilon+1)$.

Will man dem Reaktionsfeld $\vec{Z}$ Rechnung tragen, so ergibt sich aus (9) und (13)

$$
\ln k-\ln k_{0}=\frac{e_{0} \cdot z}{k T} \cdot F(\varepsilon)\left[\mu_{0} f_{0} \cdot \overline{\cos \theta_{0} / R_{0}^{2}}-\mu f \cdot \overline{\cos \theta / R^{2}}\right]
$$

mit dem Faktor $f$ des Reaktionsfeldes $8 f=\frac{(2 \varepsilon+1)\left(n^{2}+2\right)}{3\left(2 \varepsilon+n^{2}\right)}$, wobei $n$ der Brechungsindex des Esters ist. Aus Tab. 1 ist ersichtlich, daß in ausreichender Näherung $f=f_{0}$ gesetzt werden kann, so daß in Gleichung (13) an die Stelle von $F(\varepsilon)$ die Funktion

$$
F^{*}(\varepsilon)=f_{0} \cdot F(\varepsilon)
$$

zu treten hätte. 
Es ist für die Diskussion von Gleichung (13) irrelevant, ob der Mittelpunkt der Dipole $\mu_{0}$ bzw. $\mu$ auf die Peripherie des $C$-Atoms (Tab. 2 b, Spalte 2), oder in die Mitte der $C-X$.Bindung (Tab. 2 b, Spalte 3) gelegt wird.

Mit $\Varangle \widehat{k l}=\psi$ gilt

$$
\overline{\left(\cos \theta / R^{2}\right)}=\frac{1}{2 \pi} \int_{0}^{2 \pi} \cos \theta(\psi) / R^{2}(\psi) d \psi
$$

Wegen (Symbole in Abb. 2 und Tab. 2)

folgt

$$
\begin{aligned}
R^{2}(\psi) & =h^{2}+k^{2}+l^{2}-2 k l \cos \psi \\
\cos \theta(\psi) & =\left(R^{2}-B^{2}\right) / R \cdot d_{C-X} \\
B^{2} & =\left(d_{C-X}+d_{C-O} \cos 70^{\circ}\right)^{2}+l^{2}-d_{C-X}^{2} / 4
\end{aligned}
$$

$$
\overline{\left(\cos \theta / R^{2}\right)}=\left(\cos \theta / R^{2}\right)_{\psi=\frac{\pi}{2}}\left[1+\frac{3}{4}\left(\frac{k l}{R^{2}}\right)^{2} \frac{R^{2}-5 B^{2}}{R^{2}-\overline{B^{2}}}+\cdots\right]_{\psi=\frac{\pi}{2}} .
$$

In diesem Zusammenhang ist die Berücksichtigung des ersten Gliedes der Reihenentwicklung ausreichend.

Die Größen $h, k, l$ wurden aus den bekannten Bindungslängen und Bindungswinkeln ${ }^{9,10}$ bestimmt.

Diese Überlegung gilt für $\mathrm{F} \cdot, \mathrm{Cl}$., $\mathrm{Br}$ - und $\mathrm{J}$-Substitution. Bei $\mathrm{HO}$-, $\mathrm{CH}_{3} \mathrm{O}$ und $\mathrm{C}_{2} \mathrm{H}_{5} \mathrm{O}$-Substitution ist überdies freie Drehbarkeit um die $\mathrm{C}-\mathrm{O}$-Bindung berücksichtigt worden.

\section{b) Mehrfach substituierte Ester}

Im Falle der mehrfach am gleichen $C$-Atom substituierten Ester führt die vorangehend aufgezeigte Methode von (8a) ausgehend zur Gleichung

$\ln k-\ln k_{0}=\frac{e_{0} \cdot z}{k T} F^{*}(\varepsilon)\left[\sum_{\nu=1}^{3} \mu_{0}\left(\overline{\cos \theta_{0} / R_{0}^{2}}\right)_{\nu}-\sum_{\nu=1}^{3} \mu\left(\overline{\cos \theta / R^{2}}\right)_{\nu}\right]$

und, wenn in der zweiten Summe die Substituenten einander gleich sind, schließlich zu

mit

$$
\ln k-\ln k_{0}=\frac{e_{0} \cdot z}{k T} F^{*}(\varepsilon) \Delta\left[\mu\left(\overline{\cos \theta / R^{2}}\right)\right]
$$

$$
\left.\Delta\left[\mu \overline{\left(\cos \theta / R^{2}\right.}\right)\right]=\nu\left[\mu_{0}\left(\overline{\cos \theta_{0} / R_{0}^{2}}\right)-\mu\left(\overline{\cos \theta / R^{2}}\right)\right] .
$$

Mit $v=1$ enthält Gleichung (16) auch Gleichung (13).

9 A. D. Mrtcherl und L. C. Cross, Tables of Interatomic Distances and Configuration in Molecules and Ions. London 1958.

10 Landolt-Börnstein, Zahlenwerte und Funktionen, Band I, Atom- und Molekularphysik, 2. Teil, 6. Aufl., Berlin 1951. 
Der gegenseitigen Induktion der Liganden ist dadurch Rechnung getragen, daß die Dipolmomente $C-X$ aus den gemessenen Momenten der entsprechend substituierten Äthane nach üblichen Methoden ${ }^{11}$ berechnet worden sind.

\section{c) Ergebnisse}

Tab. 2 enthält alle zur Berechnung notwendigen Strukturparameter $^{9,10,12,13}$, sowie in Spalte 2 und 3 der Tab. 2 b die Gegenüberstellung von $\Delta\left[\mu\left(\overline{\cos \theta / R^{2}}\right)\right]$ mit den Meßwerten aus Tab. 1 in der

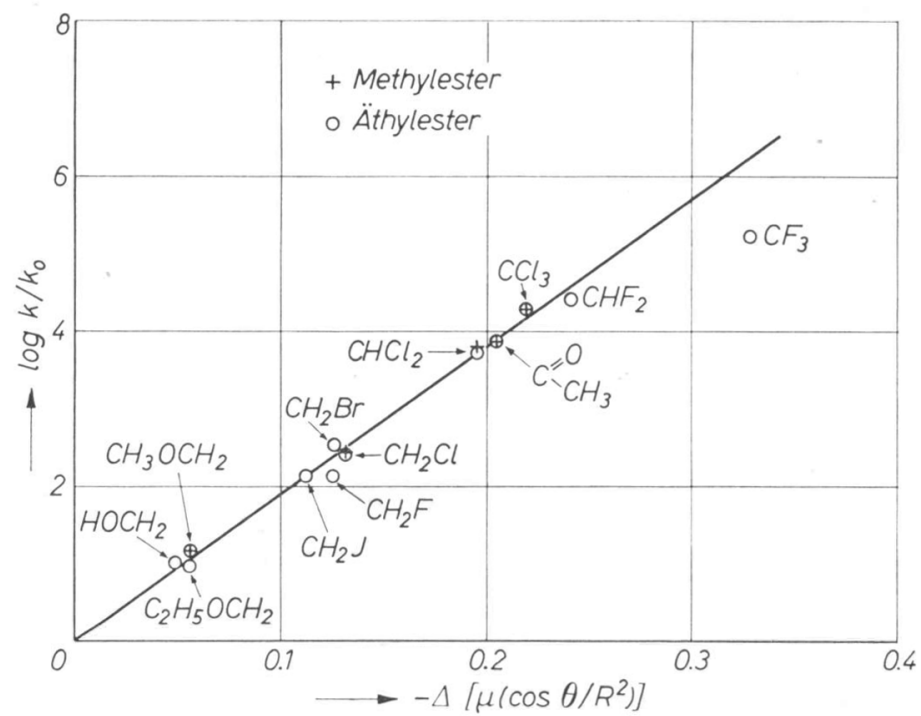

Abb. 3. Darstellung $\log \left(k / k_{0}\right)=f\left(\Delta\left[\mu\left(\overline{\cos \theta / R^{2}}\right)\right]\right)$

Form $\log \left(k / k_{0}\right)$ für neunzehn Äthyl- (Spalte 4) und Methylester (Spalte 5). Entsprechend Gleichung (16) führt die Darstellung

$$
\log \left(k / k_{0}\right)=f\left(\Delta\left[\mu\left(\overline{\cos \theta / R^{2}}\right)\right]\right)
$$

zu einer linearen Funktion (Abb.3) und zeigt damit die Gültigkeit der Annahme, daß für den gesamten Variationsbereich der Geschwindigkeitskonstanten der Hydrolyse substituierter Essigsäureester die Verschiebung aus der Änderung der elektrostatischen Wechselwirkung erklärbar ist.

11 J. R. Partington, An Advanced Treatise on Physical Chemistry, Band V, London 1954.

12 Landolt-Börnstern, Zahlenwerte und Funktionen, Band I Atom- und Molekularphysik, 3. Teil, 6. Aufl., Berlin 1951.

13 D. R. Lide, Jr., J. Amer. chem. Soc. 74 (1952) 3548. 
Substituenten- und Lösungsmitteleinfluß auf Solvolysereaktionen, I

Tabelle 2. Struktur- und Substitutionsparameter der Essigsäureester

Tabelle 2 a

\begin{tabular}{|c|c|c|c|c|c|c|}
\hline $\begin{array}{c}\text { Rest } \\
\quad R\end{array}$ & $\begin{array}{c}C-X \\
(O-X)\end{array}$ & $\begin{array}{c}d_{C-X} \\
{[\AA]}\end{array}$ & $\Varangle C C X$ & $\begin{array}{c}\mu_{-C X} \\
{[D]}\end{array}$ & $\begin{array}{r}{[R]_{\frac{\pi}{2}}} \\
{[\AA]}\end{array}$ & {$[\cos \theta]_{\frac{\pi}{2}}$} \\
\hline $\mathrm{CH}_{3}$ & $C-H$ & 1,09 & 109,5 & 0,4 & 2,63 & $-0,469$ \\
\hline $\mathrm{CH}_{2} \mathrm{OH}$ & $\begin{array}{c}O-C \\
(O-H)\end{array}$ & $\begin{array}{c}1,43 \\
(0,97)\end{array}$ & $\begin{array}{c}109 \\
(105)\end{array}$ & $\begin{array}{c}0,7 \\
(1,6)\end{array}$ & 3,15 & 0,674 \\
\hline $\mathrm{CH}_{2} \mathrm{OCH}_{3}$ & $\left(\mathrm{O}-\mathrm{CH}_{3}\right)$ & $(1,43)$ & $(105)$ & $(1,1)$ & 3,15 & 0,674 \\
\hline $\mathrm{CH}_{2} \mathrm{OC}_{2} \mathrm{H}_{5}$ & $\left(\mathrm{O}-\mathrm{C}_{2} \mathrm{H}_{5}\right)$ & $(1,43)$ & (105) & $(1,1)$ & 3,15 & 0,674 \\
\hline $\mathrm{CH}_{2} \mathrm{~F}$ & $F-C$ & 1,39 & 109,5 & 1,41 & 2,71 & 0,516 \\
\hline $\mathrm{CHF}_{2}$ & $F-C$ & 1,35 & 110 & 1,34 & 2,70 & 0,506 \\
\hline$C F_{3}$ & $F-C$ & 1,33 & 110 & 1,19 & 2,69 & 0,503 \\
\hline $\mathrm{CH}_{2} \mathrm{Cl}$ & $C l-C$ & 1,77 & 109,5 & 1,46 & 2,81 & 0,561 \\
\hline $\mathrm{CHCl}_{2}$ & $C l-C$ & 1,77 & 110 & 1,00 & 2,81 & 0,561 \\
\hline$C C l_{3}$ & $C l-C$ & 1,77 & 109 & 0,65 & 2,81 & 0,561 \\
\hline $\mathrm{CH}_{2} \mathrm{Br}$ & $B r-C$ & 1,93 & 109,5 & 1,39 & 2,85 & 0,580 \\
\hline $\mathrm{CH}_{2} \mathrm{~J}$ & $J-C$ & 2,13 & 109,5 & 1,21 & 2,91 & 0,602 \\
\hline$C^{O}$ & $O=C$ & 1,24 & 120 & 2,5 & 2,42 & 0,830 \\
\hline $\mathrm{CH}_{3}$ & $\mathrm{C}-\mathrm{CH}_{3}$ & 1,54 & 120 & 0,4 & & \\
\hline Periph. $C$ & $C$ & 0,77 & 109,5 & & 2,74 & 0,533 \\
\hline
\end{tabular}

Tabelle 2b

\begin{tabular}{|c|c|c|c|c|}
\hline \multirow{2}{*}{$\begin{array}{c}\text { Rest } \\
\quad R\end{array}$} & \multicolumn{2}{|c|}{$\Delta\left[\mu\left(\overline{\cos \theta / R^{2}}\right)\right]$} & \multicolumn{2}{|c|}{$\log \left(k / k_{0}\right)$} \\
\hline & Periph. & Dipolmitte & $R^{\prime}=C_{2} H_{5}$ & $R^{\prime}=C H_{3}$ \\
\hline $\mathrm{CH}_{2} \mathrm{OH}$ & $-0,049$ & $-0,047$ & 1,054 & \\
\hline $\mathrm{CH}_{2} \mathrm{OCH}_{3}$ & $-0,058$ & $-0,056$ & 1,129 & 1,158 \\
\hline $\mathrm{CH}_{2} \mathrm{OC}_{2} \mathrm{H}_{5}$ & $-0,058$ & $-0,056$ & 1,023 & \\
\hline $\mathrm{CH}_{2} \mathrm{~F}$ & $-0,128$ & $-0,126$ & 2,138 & \\
\hline $\mathrm{CHF}_{2}$ & $-0,245$ & $-0,241$ & 4,419 & \\
\hline$C F_{3}$ & $-0,336$ & $-0,329$ & 5,215 & \\
\hline $\mathrm{CH}_{2} \mathrm{Cl}$ & $-0,131$ & $-0,131$ & 2,401 & 2,432 \\
\hline $\mathrm{CHCl}_{2}$ & $-0,197$ & $-0,196$ & 3,683 & 3,806 \\
\hline$C C l_{3}$ & $-0,222$ & $-0,220$ & 4,252 & 4,291 \\
\hline $\mathrm{CH}_{2} \mathrm{Br}$ & $-0,126$ & $-0,126$ & 2,524 & \\
\hline $\mathrm{CH}_{2} \mathrm{~J}$ & $-0,114$ & $-0,113$ & 2,123 & \\
\hline$C(=O) C H_{3}$ & & $-0,205$ & $3,845^{1}$ & $3,852^{1}$ \\
\hline
\end{tabular}


Tabelle 3. Aktivierungsgrößen der basischen Esterhydrolyse

\begin{tabular}{l|c|c|c}
\hline \multicolumn{1}{c|}{ Ester } & $\begin{array}{c}\Delta G_{298}^{\ddagger} \\
{[\mathrm{kcal} / \mathrm{Mol}]}\end{array}$ & $\begin{array}{c}\Delta H_{298}^{+} \\
\text {[kcal/Mol] }\end{array}$ & $\begin{array}{c}-\Delta S_{998}^{ \pm} \\
\text {[Cl/Mol] }\end{array}$ \\
\hline $\mathrm{CH}_{3} \mathrm{CO}_{2} \mathrm{CH}_{3}$ & 18,48 & 11,61 & 22,6 \\
$\mathrm{CH}_{3} \mathrm{OCH}_{2} \mathrm{CO}_{2} \mathrm{CH}_{3}$ & 16,83 & 9,62 & 22,2 \\
$\mathrm{ClCH}_{2} \mathrm{CO}_{2} \mathrm{CH}_{3}$ & 15,12 & 9,18 & 19,9 \\
$\mathrm{Cl}_{2} \mathrm{CHCO}_{2} \mathrm{CH}_{3}$ & 13,25 & 7,50 & 19,3 \\
$\mathrm{Cl}_{3} \mathrm{CCO}_{2} \mathrm{CH}_{3}$ & 12,58 & 7,22 & 22,9 \\
$\mathrm{CH}_{3} \mathrm{CO}_{2} \mathrm{C}_{2} \mathrm{H}_{5}$ & 18,79 & 11,26 & 25,2 \\
$\mathrm{HOCH}_{2} \mathrm{CO}_{2} \mathrm{C}_{2} \mathrm{H}_{5}$ & 17,31 & 10,65 & 22,3 \\
$\mathrm{CH}_{3} \mathrm{OCH}_{2} \mathrm{CO}_{2} \mathrm{C}_{2} \mathrm{H}_{5}$ & 17,22 & 9,20 & 26,9 \\
$\mathrm{C}_{2} \mathrm{H}_{5} \mathrm{OCH}_{2} \mathrm{CO}_{2} \mathrm{C}_{2} \mathrm{H}_{5}$ & 17,49 & 9,34 & 26,8 \\
$\mathrm{FCH}_{2} \mathrm{CO}_{2} \mathrm{C}_{2} \mathrm{H}_{5}$ & 15,80 & 9,60 & 20,8 \\
$\mathrm{~F}_{2} \mathrm{CHCO}_{2} \mathrm{C}_{2} \mathrm{H}_{5}$ & 12,72 & 7,29 & 18,2 \\
$\mathrm{~F}_{3} \mathrm{CCO}_{2} \mathrm{C}_{2} \mathrm{H}_{5}$ & 11,64 & 8,11 & 13,5 \\
$\mathrm{ClCH}_{2} \mathrm{CO}_{2} \mathrm{C}_{2} \mathrm{H}_{5}$ & 15,46 & 8,69 & 22,7 \\
$\mathrm{Cl}_{2} \mathrm{CHCO}_{2} \mathrm{C}_{2} \mathrm{H}_{5}$ & 13,72 & 6,90 & 22,9 \\
$\mathrm{Cl}_{3} \mathrm{CCO}_{2} \mathrm{C}_{2} \mathrm{H}_{5}$ & 12,95 & 6,79 & 20,7 \\
$\mathrm{BrCH}_{2} \mathrm{CO}_{2} \mathrm{C}_{2} \mathrm{H}_{5}$ & 15,30 & 8,26 & 23,6 \\
$\mathrm{JCH}_{2} \mathrm{CO}_{2} \mathrm{C}_{2} \mathrm{H}_{5}$ & 15,83 & 7,99 & 26,3
\end{tabular}

Der Anstieg dieser linearen Funktion ist ein Maß für $F^{*}(\varepsilon)$ und kann zur Untersuchung des Lösungsmitteleinflusses herangezogen werden.

Tab. 3 enthält die experimentell bestimmten Aktivierungsgrößen $\Delta G^{\ddagger}, \Delta H^{\ddagger}$ und $\Delta S^{\ddagger}$. Ein einfacher Zusammenhang zwischen $\Delta H^{\ddagger}$ bzw. $\Delta S^{\ddagger}$ und dem Substituentenparameter $\Delta\left[\mu\left(\overline{\cos \theta / R^{2}}\right)\right]$ ist nur in Reihen gegeben, in denen Lösungsmitteleinfluß und sterischer Effekt vergleichbar sind.

Wir danken dem Direktor des Institutes für Physikalische Chemie, Herrn Professor Dr. N. G. SchmahL, für das dieser Arbeit entgegengebrachte Interesse. Der deutschen Forschungsgemeinschaft danken wir für die Förderung dieser Arbeit durch die von ihr gewährte Sachbeihilfe. 